Re-impressão de artigo publicado na Acta Amazonica, 23(2/3):181-185, tendo em vista o grande número de erros de impressão ocorridos.

\title{
PRENYLATED XANTHONES FROM Rheedia acuminata
}

\author{
Maria das G. B. ZOGHBI ${ }^{1}$, Patrícia D. de D. B. LIMA, Maria do P. Socorro C. \\ da CUNHA ${ }^{1}$, Angelo da C. PINTO
}

\begin{abstract}
Pyranojacareubin; 1,5-dihydroxy-6',6'-dimethyl-2H-pyran(2',3':6,7) -6",6"'-dimethyl-2H,4H-pyran (2", 3":2,3)xanthone and a new xanthone 1,6-dihydroxy-5-methoxy-6',6'-dimethyl-2H-pyran (2', 3':3,2)-7-(3,3-dimethylprop-2-enyl)xanthone were isolated from the ether extract of the root bark of Rheedia acuminata together with friedelin and friedelanol.
\end{abstract}

Key words: Guttiferae, Rheedia, Xanthones.

Xantonas Preniladas de Rheedia acuminata (GUTTIFERAE)

RESUMO - Piranojacareubina; 1,5-diidroxi-6',6'-dimetil-2H-pirano(2',3':6,7)-6",6"'-dimetil$2 \mathrm{H}, 4 \mathrm{H}\left(2^{\prime \prime}, 3^{\prime \prime}: 2,3\right)$ xantona e uma xantona inédita 1,6-diidroxi-5-metoxi-6',6'-dimetil-2Hpirano(2',3':3,2)-7-(3,3-dimetilprop-2-enil) xantona foram isoladas do extrato etéreo da casca da raiz de Rheedia acuminata além de friedelina e friedelanol.

Palavras-chave: Guttiferae, Rheedia, Xantonas.

\section{INTRODUCTION}

The family Guttiferae numbers over 1000 species, which occur widely in temperate regions. Xanthones or the related benzophenones have been found in all their major and several minor genera (BENNETT \& LEE, 1989). Prenylated xanthones are widely distributed in the Guttiferae, and the genus Rheedia has been shown to be rich with them. They have been isolated from the $R$. benthamiana (DELLE MONACHE et al., 1981), $R$. gardneriana (DELLE MONACHE $e t$ al., 1983) and R. brasiliensis (DELLE MONACHE et al., 1984); we now report the isolation of three prenylated xanthones from the $R$. acuminata, which are present in the ether extract of the roots. Two xanthones were iden- tified as pyranojacareubin (1) and 1,5dihydroxy-6',6'-dimethyl-2H-py$\operatorname{ran}\left(2^{\prime}, 3^{\prime}: 6,7\right)-6^{\prime \prime}, 6^{\prime \prime}$-dimethyl-2H,4Hpyran (2", $\left.3^{\prime \prime}: 2,3\right)$ - xanthone (2), while the third one, (3), was novel and was named acuminatine. The triterpenes friedelin and friedelanol also were isolated.

\section{Identification of Constituents}

Compound 1, yellow needles (hexane-acetone), mp 261-263", [lit. 259.5-260.5" (Et 20$)$ ], $\mathrm{C}_{23} \mathrm{H}_{20} \mathrm{O}_{6}\left(\mathrm{M}^{+}\right.$at $\mathrm{m} / \mathrm{z}$ 392) two 2,2-dimethyl-2H-pyran rings and two separated aromatic protons ('H NMR evidence) exhibited IR and MS spectra data, which were in agreement with those reported in the literature for pyranojacareubin (DELLE MONACHE et al., 1984). Hydrogenation of 1 furnished 4 (mp

I Instituto Nacional de Pesquisas da Amazônia, Coordenação de Pesquisas em Produtos Naturais, Caixa Postal 478, 69011-970 - Manaus, Amazonas, Brasil.

2 Instituto de Química, Universidade Federal do Rio de Janeiro, Caixa Postal 1573, 20001-970, Rio de Janeiro, RJ, Brasil. 
234-235").

Compound 2, yellow crystals (hexane-acetone), mp 212-215" [lit. 202$\left.204^{\prime \prime}\right], \mathrm{C}_{23} \mathrm{H}_{22} \mathrm{O}_{6}$ at $\mathrm{m} / \mathrm{z}$ 394, was isolated in a mixture with pyranojacareubin. The 'H NMR spectrum showed beside signals of the pyranojacareubin system, two triplets $(\delta 2.90,2 \mathrm{H}, \mathrm{J}=7.0 \mathrm{~Hz}$ and $1.91,2 \mathrm{H}, \mathrm{J}=7.0 \mathrm{~Hz}$ ) that were assigned to $\mathrm{H}-4$ ' and $\mathrm{H}-5$ ', respectively (DELLE MONACHE et al., 1984). The ${ }^{13} \mathrm{C}$ NMR chemical shifts are presented in Table 1, along with reported shifts for the closely related geronxanthone B (CHANG et al., 1989). The chemical shifts were assigned on the basis of the PND and DEPT spectra. The presence of the two carbonyl groups ( $\delta 180.4$ and $180.1)$ and two methylenic carbons $(\delta$ 32.5 and 21.7) and the signals peaks at $394\left(\mathrm{M}^{+}\right), 379\left(\mathrm{M}^{+}-\mathrm{Me}\right)$ and $323\left(\mathrm{M}^{+}-\right.$ Me- $\left.\mathrm{C}_{4} \mathrm{H}_{8}\right)$ in the mass spectrum permitted identification of the 1,5-dihydroxy6',6'-dimethyl-2H-pyran(2',3': 6,7)-6", 6"-dimethyl-2H, 4H(2",3": 2,3)xanthone.

Compound 3 , yellow crystals (hexane-Et ${ }_{2} \mathrm{O}, \mathrm{mp} 152-154^{\prime \prime}, \mathrm{C}_{24} \mathrm{H}_{24} \mathrm{O}_{6}$ $\left(\mathrm{M}^{+}\right.$at $\left.\mathrm{m} / \mathrm{z} 408\right)$ showed characteristic UV and IR spectra of a xanthone.

Table 1. ${ }^{13} \mathrm{C}$ NMR chemical shifts of 1,2 and 3 , in $\mathrm{CDCl}_{3}$

\begin{tabular}{|c|c|c|c|c|c|}
\hline & 1 & 2 & ref.6 & 3 & ref. 8 \\
\hline C-1 & 157.6 & 157.6 & 163.1 & 157.8 & 157.8 \\
\hline C-2 & 104.4 & 113.4 & 113.5 & 104.7 & 104.4 \\
\hline C-3 & 160.3 & 156.7 & 161.8 & 160.3 & 159.8 \\
\hline C-4 & 95.2 & 95.2 & 95.7 & 95.0 & 94.0 \\
\hline C-4a & 156.7\# & 157.0\# & 155.8 & 156.6 & 156.1 \\
\hline$C-4 b$ & $144.7 \#$ & $142.8 \#$ & 144.7 & 147.8 & 155.6 \\
\hline C-5 & 132.2 & 132.2 & 131.7 & 133.9 & 101.6 \\
\hline$C-6$ & 145.0\# & $146.4 \#$ & 144.9 & 152.6 & 154.5 \\
\hline C-7 & $117.7 \#$ & $118.1 \#$ & 117.6 & 125.6 & 142.7 \\
\hline C-8 & 121.2 & 121.2 & 121.4 & 120.5 & 136.9 \\
\hline C-8a & 114.5 & 114.5 & 113.6 & 114.0 & 112.1 \\
\hline$C-8 b$ & 103.1 & 103.1 & 103.8 & 103.1 & 103.6 \\
\hline C-9 & $180.4 \#$ & $180.1 \#$ & 180.5 & 180.1 & 181.8 \\
\hline C-4' & 127.3 & 32.5 & - & 127.4 & 126.9 \\
\hline C-5' & 116.1 & 21.7 & -- & 115.5 & 115.6 \\
\hline C-1" & - & - & -. & 25.8 & 26.5 \\
\hline C-2" & -- & -- & -- & 121.0 & 123.1 \\
\hline C-3" & -- & -. & -. & 133.2 & 131.8 \\
\hline C-4" & 127.5 & 127.5 & 114.5 & 17.8 & 18.1 \\
\hline C-5" & $115.4 \#$ & $113.8 \#$ & 130.8 & 28.1 & 25.6 \\
\hline$C-6^{\prime}$ & 78.8\# & $78.0 \#$ & -- & 78.2 & 77.8 \\
\hline C-6" & 78.1 & 77.1\# & 78.6 & - & - \\
\hline 6'-Me & 28.4 & 26.9\# & - & 28.4 & 28.3 \\
\hline 6"-Me & 28.3 & 28.2 & 28.4 & - & - \\
\hline $\begin{array}{l}\text { C-3"' } \\
\text { C-3a", }\end{array}$ & 二- & - & $\begin{array}{l}40.9 \\
26.9\end{array}$ & 二 & - \\
\hline C-4'"' & - & - & 149.6 & - & - \\
\hline C-5"' & -. & - & 113.7 & -- & - \\
\hline $\mathrm{OCH}_{3}$ & -. & -- & - & 61.8 & 61.8 \\
\hline
\end{tabular}

Assigned by comparison with the cyclo derivative of theediaxanthone B (DELLE MONACHE et al., 1981)

\# Assigned may be interchanged 
The chelated hydroxyl and C-6 hydroxyl, which were indicated by typica UV spectrum, underwent modifications with additives (MESQUITA et al., 1968). The presence of the free hydroxyl was confirmed by methylation with diazomethane $\left(\mathrm{M}^{+}\right.$at $\left.\mathrm{m} / \mathrm{z} 422\right)$. The $\gamma, \gamma$-dimethylallyl and 2,2dimethylchromene groups were characterized by ${ }^{1} \mathrm{H}$ NMR data. The signal at $\delta 13.50$ confirmed the chelated hydroxyl. The localization of the chromene group at C-2 and C- 3 and C1 hydroxyl was established by comparison of their ${ }^{1} \mathrm{H}$ and ${ }^{13} \mathrm{C}$ NMR data (Table 1) with reported chemical shifts for the closely related compound (SEN et al., 1980). The signal of the $\mathrm{OCH}_{3}$ group in the ${ }^{13} \mathrm{C}$ NMR at $\delta 61.8$ indicates that it is bonded to C-5, between two ortho groups (CHAUDHURI et al., 1978). The localization of the $\gamma, \gamma-$ dimethyallyl at C-7 was confirmed by the long range $\left(\mathrm{J}^{3}\right)$ coupling, and NOE experiments.

\section{EXPERIMENTAL}

\section{General Experimental Procedure}

All melting points were uncorrected. UV spectra were recorded on a Perkin Elmer 402 and IR spectra on a Perkin-Elmer 298 spectrophotometer, ${ }^{13} \mathrm{C}$ NMR spectra on a Bruker spectrometer operating at $50.0 \mathrm{MHz}$ and 'H NMR spectra on a Bruker spectrometer operating at 100 and 200 $\mathrm{MHz} .{ }^{13} \mathrm{C}$ NMR and ${ }^{1} \mathrm{H}$ NMR spectra were measured with tetramethylsilane (TMS) as internal reference. Mass spectra were recorded on a HP-5987A instrument at $70 \mathrm{eV}$.

\section{PLANT MATERIAL}

The root bark of $R$. acuminata was collected in Araguacema, Goiás State, Brazil, and identified by the botanist Dr. William A. Rodrigues, INPA, Manaus, Brazil.

\section{EXTRACTION AND ISOLA- TION OF CONSTITUENTS}

The root bark $(3.05 \mathrm{~kg})$ was reduced to saw dust and extracted at room temperature with petrol ether. The solvent was evaporated giving $94.2 \mathrm{~g}$ of the extract. Part of it $(10.0 \mathrm{~g})$ was chromatographed on silica giving the following compounds: $1,2,3$ friedelin and friedelanol, eluted with hexane/acetone $4 \% ; 1(13.0 \mathrm{mg})$ was recrystallized from hexane-acetone $(1: 1) ; 2(8.0 \mathrm{mg})$ was purified by CCCP; $3(60.8 \mathrm{mg})$ was recrystallized from hexane-diethyl ether $(1: 1)$.

\section{Spectroscopy data of Constituents}

1,5-dihydroxy-6',6'-dimethyl-2Hpyran $\left(2^{\prime}, 3^{\prime}: 3,2\right)-6^{\prime \prime}, 6^{\prime \prime}$-dimethyl-2Hpyran $(2 ", 3 ": 6,7) x$ anthone (pyranojacareubin, 1), $\mathrm{C}_{23} \mathrm{H}_{20} \mathrm{O}$, $\mathrm{mp}$ 261-263 ${ }^{\circ}$ (yellow needles). IR v $\mathrm{KBr}$ $\max \left(\mathrm{cm}^{-1}\right): 3480,2969,1638,1605$, $1467,1374,1199,1157 .{ }^{~ ' H ~ N M R ~}$ $\left(\mathrm{CDCl}_{3}: \delta 13.80(1 \mathrm{H}, 1-\mathrm{OH}), 7.48\right.$ $(1 \mathrm{H}, \mathrm{s}, \mathrm{H}-8), 6.73(1 \mathrm{H}, \mathrm{d}, \mathrm{J}=10.0 \mathrm{~Hz}$, H-4'), $6.44(1 \mathrm{H}, \mathrm{s}, \mathrm{H}-4), 6.45(1 \mathrm{H}, \mathrm{d}$, $\mathrm{J}=10.0 \mathrm{~Hz}, \mathrm{H}-4 "), 5.74(1 \mathrm{H}, \mathrm{d}, \mathrm{J}=10.0$ $\mathrm{Hz}, \mathrm{H}-5 "), 5.60(1 \mathrm{H}, \mathrm{d}, \mathrm{J}=10.0 \mathrm{~Hz}, \mathrm{H}-$ $\left.5^{\prime}\right), 1.55+1.45(6 \mathrm{H}+6 \mathrm{H}, \mathrm{s}, 4 \times \mathrm{Me})$. MS m/z (rel. int.): $392\left[\right.$ [M] $^{+}$(18), 377 
[M-Me] $^{+}(100), 323$ (14), 267 (1), 181 $[\mathrm{M}-\mathrm{Me}-\mathrm{Me}]^{2+}(24)$.

1,5-dihydroxy-6',6'-dimethyl2H-pyran(2', 3':6,7)-6", 6"-dimethyl$2 \mathrm{H}, 4 \mathrm{H}-$ pyran $\left(2^{\prime \prime}, 3^{\prime \prime}: 3,2\right)$ xanthone (dihydropyranojacareubin, $\mathrm{C}_{23} \mathrm{H}_{22} \mathrm{O}_{6}$, mp 212-215 (yellow needles). 'H NMR $\left(\mathrm{CDCl}_{3}\right): \delta 13.8$ $(1 \mathrm{H}, \mathrm{s}, 1-\mathrm{OH}), 7.48(1 \mathrm{H}, \mathrm{s}, \mathrm{H}-8)$, $6.45(1 \mathrm{H}, \mathrm{d}, \mathrm{J}=10.0 \mathrm{~Hz}, \mathrm{H}-4 "), 6.44$ $(1 \mathrm{H}, \mathrm{s}, \mathrm{H}-4), 5.74(1 \mathrm{H}, \mathrm{d}, \mathrm{J}=10.0 \mathrm{~Hz}$, H-5"), 2.90 (2H, t, J=7.0 Hz, H-4'), $1.91\left(2 \mathrm{H}, \mathrm{t}, \mathrm{J}=7.0 \mathrm{~Hz}, \mathrm{H}-5^{\prime}\right), 1.55+$ $1.45(6 \mathrm{H}+6 \mathrm{H}, \mathrm{s}, 4 \times \mathrm{Me}) . \mathrm{MS} \mathrm{m} / \mathrm{z}$ (rel. int.): $394\left[^{\mathrm{M}^{+}}\right.$(12), 379 (44), 323 (15)

Hydrogenation of 2: Compound 2 $(62.0 \mathrm{mg})$ was hydrogenated by the usual method giving as a pale yellow needles, mp 234-235 (methanol-hexane). IR $v \max \left(\mathrm{KBr}, \mathrm{cm}^{-1}\right): 3420,2920$, $1630,1600,1580,1440,1150,870,820$. ' $\mathrm{H}$ NMR $\left(\mathrm{CDCl}_{3}\right): \delta 7.45(1 \mathrm{H}, \mathrm{s}, \mathrm{H}-8)$, $6.34(1 \mathrm{H}, \mathrm{s}, \mathrm{H}-4), 2.84(2 \mathrm{H}, \mathrm{t}, \mathrm{J}=6.2 \mathrm{~Hz}$, H-4'), 2.65 (2H, t, J=6.6 Hz, H-4"), 1.83 $\left(2 \mathrm{H}, \mathrm{t}, \mathrm{J}=6.2 \mathrm{~Hz}, \mathrm{H}-5^{\prime}\right), 1.78(2 \mathrm{H}, \mathrm{t}$, $\mathrm{J}=6.6 \mathrm{~Hz}, \mathrm{H}-5 "), 1.37$ (3H, s, Me), 1.31 $(3 \mathrm{H}, \mathrm{s}, \mathrm{Me})$.

1,6-dihydroxy-5-methoxy-6',6'dimethyl-2H-pyran (2', 3':3,2)-7-(3,3dimethylprop-2-enyl) xanthone (acumi-

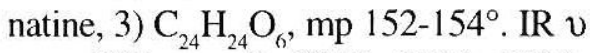
$\max \left(\mathrm{KBr}, \mathrm{cm}^{-1}\right): 3350,2930,2890$, $2840,1650,1580,1460,1350,1190$, $1150,810,770$. UV $\lambda \max (\mathrm{EtOH}, \mathrm{nm})$ (log $\varepsilon): 244(4.79), 279$ (4.83), 289 (4.83), 334, (4.60); $\lambda \max \left(\mathrm{AlCl}_{3}, \mathrm{~nm}\right)$ (after 30 min.): 243, 289, 358; $\lambda$ max $(\mathrm{HCl}, \mathrm{nm}): 246,296,358 ; \lambda \max$ $(\mathrm{NaOH}, \mathrm{nm}): 247,280,316 \mathrm{sh}, 386 ; \lambda$ $\max (\mathrm{HCl}, \mathrm{nm}): 243,280,334 ; \lambda \max$
(NaOAc, nm): 244, 279, 380; $\lambda$ max $\left(\mathrm{H}_{3} \mathrm{BO}_{3}, \mathrm{~nm}\right): 244,289,334$. 'H NMR $\left(\mathrm{CDCl}_{3}\right): \delta 13.50(1 \mathrm{H}, \mathrm{s}, 1-\mathrm{OH}), 7.73(1 \mathrm{H}$, $\mathrm{s}, \mathrm{H}-8), 6.72\left(1 \mathrm{H}, \mathrm{d}, \mathrm{J}=10.0 \mathrm{~Hz}, \mathrm{H}-4^{\prime}\right), 6.58$ $(1 \mathrm{H}, \mathrm{s}, 6-\mathrm{OH}), 6.37(1 \mathrm{H}, \mathrm{s}, \mathrm{H}-4), 5.59(1 \mathrm{H}$, d, J $\left.=10.0 \mathrm{~Hz}, \mathrm{H}-5^{\prime}\right), 5.35(1 \mathrm{H}, \mathrm{t}, \mathrm{J}=7.0 \mathrm{~Hz}$, $\mathrm{H}-2$ "), 4.10 (3H, s, OMe), $3.40(2 \mathrm{H}, \mathrm{d}$, $\mathrm{J}=7.0 \mathrm{~Hz}, \mathrm{H}-1 "), 1,76(3 \mathrm{H}, \mathrm{s}, \mathrm{Me}), 1,74$ $(3 \mathrm{H}, \mathrm{s}, \mathrm{Me}), 1.50(6 \mathrm{H}, \mathrm{s}, 2 \times \mathrm{Me})$. MS m/ z (rel. int.): $408[\mathrm{M}]^{+}(28), 393$ [M-Me] $^{+}$ (100), 377 (5), 361 (5), 355 (3), 335 (10), $323(5), 189$ (6), 182 (2), 174 (3), 169 (5), 38 (69).

Mono-O-methyl ether of 3. Compound $3(14.0 \mathrm{mg})$ was suspended in $\mathrm{Et}_{2} \mathrm{O}$, treated with excess $\mathrm{CH}_{2} \mathrm{~N}_{2}$ and left overnight. The solvent was evaporated and the product was recrystallized from haxanediethyl ether (14.4 mg) mp 135-138 . IR $v \max \left(\mathrm{KBr}, \mathrm{cm}^{-1}\right): 2980,2920,1650$, $1600,1570,1420,1160,830$. MS m/z (rel. int.): $422[\mathrm{M}]^{+}(26), 407$ [M-Me] (100), 393 (13), 377 (3), 361 (2), 349 (3), 335 (2), 323 (7), 196 (9).

\section{RESULTS AND DISCUSSION}

From the ether extract of the root bark of Rheedia acuminata were isolated, by chromatographic techniques pyranojacareubin; 1,5-dihydroxy-6',6'dimethyl-2H-pyran(2',3':6,7)6",6"d i m e t h y l- $2 \mathrm{H}, 4 \mathrm{H}-$ pyran $(2 ", 3 ": 2,3) x a n t h o n e$, a new xanthone 1,6-dihydroxy-5-methoxy-6',6'dimethyl-2H-pyran (2', $\left.3^{\prime}: 3,2\right)-7-(3,3-$ dimethylprop-2-enyl) xanthone together with friedelin and friedelanol. The identification or structural elucidation of the compounds were based on spectroscopy techniques. 


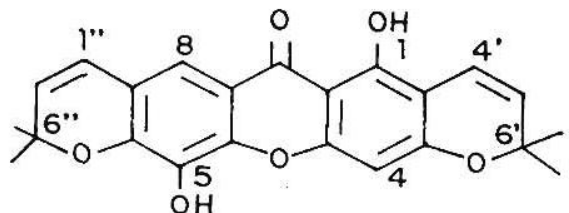

(1)<smiles>COc1c(O)c(C[18C]=C(C)C)cc2c(=O)c3c(O)c4c(cc3oc12)OC(C)(C)C=C4</smiles>

\section{Literature Cited}

BENNETT, G. J.; LEE, H. H. 1989. Xanthones from Guttiferae. Phytochemistry, 28(4), 967.

CHANG, C. H; LIN, C. C.; HATTORI, M; NAMBA, T. 1989. Four prenylated xanthones from Cudrania cochinchinensis. Phytochemistry, 28(2):595.

CHAUDHURI, R. K.; ZYMALKOWSKI, F.; FRAHM, A. W. 1978, ${ }^{13} \mathrm{C}$ NMR-Spectroscopy of Polymethoxyxanthones. Tetrahedron, 34:1837.

DELLE MONACHE, F; BOTTA, B; NICOLETTI, M.; BARROS COELHO, J. S. de; ANDRADE LYRA, F. D. de. 1981. Three new xanthones and macluraxanthone from Rheedia benthamiana Pl. Triana (Guttiferae). $J$. Chem. Soc. Perkin Trans., 1, 484.

DELLE MONACHE, G.; DELLE MONACHE, : F.; MARINI BETTOLO, G. B.; ALVES DE LIMA, R. 1983. Chemical investigation of the genus Rheedia. II. Prenylated xanthones from Rheedia gardneriana. $J$. Nat. Prod., 46, 655.<smiles>CC1(C)C=Cc2c(cc3oc4c(O)c5c(cc4c(=O)c3c2O)CCC(C)(C)O5)O1</smiles>

(2)<smiles></smiles>

(4)

DELLE MONACHE, G.; BOTTA, B.; MELLO, J. F., COELHO, J. S. de B.; MENICHINI, F. 1984. Chemical investigation of the genus Rheedia. IV. Three new xanthones from Rheedia brasiliensis. J. Nat. Prod., 47(4): 620.

DELLE MONACHE, G.; DELLE MONACHE, F.; WATERMAN, P. G.; CRICHTON, E. G.; LIMA, R. A. de 1984. Minor xanthones from Rheedia gardneriana. Phytochemistry, 23(8): 1757.

MESQUITA, A. A. L.; CORREA, D. e B.; GOTTLIEB, O. R.; MAGALHÃES, M. T. 1968. Métodos para investigação estrutural de xantonas. II. Espectroscopia no ultra-violeta e no visível. An. Acad. Brasil. Ciênc., 40(2):167.

SEN, A. K.; SARKAR, K. K.; MAZUMDER, P. C.; BARNETI, N.; UUSVUORI, R.; HASE, T. A. 1980. A xanthone from Garcinia mangostana. Phytochemistry, 19:2223. 